\title{
Ptychographic x-ray imaging of surfaces on crystal truncation rod
}

\author{
Chenhui Zhu, ${ }^{1, a)}$ Ross Harder, ${ }^{2}$ Ana Diaz, ${ }^{3}$ Vladimir Komanicky, ${ }^{4}$ Andi Barbour, ${ }^{1}$ \\ Ruqing Xu, ${ }^{2}$ Xiaojing Huang, ${ }^{5}$ Yaohua Liu, ${ }^{1}$ Michael S. Pierce, ${ }^{6}$ Andreas Menzel, ${ }^{3}$ \\ and Hoydoo You ${ }^{1, b)}$ \\ ${ }^{1}$ Materials Science Division, Argonne National Laboratory, Argonne, Illinois 60439, USA \\ ${ }^{2}$ Advanced Photon Source, Argonne National Laboratory, Argonne, Illinois 60439, USA \\ ${ }^{3}$ Paul Scherrer Institut, Villigen PSI 5232, Switzerland \\ ${ }^{4}$ Faculty of Sciences, Safarik University, Kosice 04154, Slovakia \\ ${ }^{5}$ National Synchrotron Light Source II, Brookhaven National Laboratory, Upton, New York 11973, USA \\ ${ }^{6}$ School of Physics and Astronomy, Rochester Institute of Technology, Rochester, New York 14623, USA
}

(Received 15 July 2014; accepted 22 February 2015; published online 12 March 2015)

\begin{abstract}
Ptychography is a high-resolution imaging technique, which does not require lenses for image magnification and which provides phase contrast with high sensitivity. Here, we propose to use $\mathrm{X}$-ray ptychography for the imaging of surface structure in crystalline samples. We show that ptychography can be used to image atomic step structures using coherent diffraction patterns recorded along the crystal truncation rod of a crystal surface. In a proof-of-concept experiment on a Pt (111) sample, we present ptychographic reconstructions showing features consistent with surface steps. Due to the penetration power of x-rays, this method could find interesting applications for the study of surface structures under buried interfaces or in harsh environments. (c) 2015 AIP Publishing LLC. [http://dx.doi.org/10.1063/1.4914927]
\end{abstract}

Imaging surface structure is of high relevance for understanding interfacial reactivity processes such as those occurring during catalysis ${ }^{1}$ or electrocatalysis. ${ }^{2}$ However, typical techniques to directly image surface topology on an atomic scale, such as scanning tunneling microscopy, ${ }^{3}$ atomic force microscopy, ${ }^{4}$ or electron microscopy, ${ }^{5}$ often fail to investigate systems in situ at high temperatures, under highly reacting conditions, or under solid or liquid solvents.

$\mathrm{X}$-rays offer a good opportunity to study systems in reactive environments or buried interfaces due to their large penetration depth. In particular, crystal truncation rods (CTRs) technique is sensitive to surface structure on the atomic scale, ${ }^{6}$ and has been used for buried interfaces such as electrode-electrolyte interfaces, ${ }^{7}$ nanofacets, ${ }^{8}$ and lightelement overlayers. ${ }^{9}$ The applicability of CTR can be elevated to a new level by using coherent $\mathrm{x}$-rays. Diffraction patterns are speckled and sensitive to spatial and temporal details of the surface illuminated. For example, the spatial sensitivity was used in reconstructions of surface steps by Vartanyants et al., ${ }^{10}$ and the temporal sensitivity was used to study dynamics of surface steps and domains by Pierce et al. ${ }^{11}$ Reflection x-ray imaging has also been performed to achieve one-dimensional atomic height images, ${ }^{12}$ two dimensional lithographic images ${ }^{13}$ and full-field images with a magnifying lens. ${ }^{14}$

Ptychography is a recently developed coherent diffraction imaging (CDI) technique. Coherent diffraction patterns are recorded in the far field at partially overlapping regions of the specimen and phase retrieval algorithms are then used to reconstruct the complex-valued transmissivity of the specimen. ${ }^{15,16}$ Here, we propose $x$-ray ptychography in

\footnotetext{
${ }^{a)}$ Present address: Advanced Light Source, Lawrence Berkeley National Lab, 1 Cyclotron Road, Berkeley, California 94720, USA.

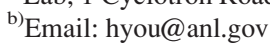

reflection geometry at or near an anti-Bragg along a specular CTR to image surface steps with high phase sensitivity, providing sufficient space without lenses around the specimen for environmental requirements. Our approach is similar to the recently developed Bragg ptychography ${ }^{17,18}$ with respect to the use of large angle scattering. However, it is complementary because the Bragg ptychography is primarily sensitive to strain fields, while our approach is primarily sensitive to the surface steps as well as to surface reconstructions ${ }^{19}$ or step edge relaxation. ${ }^{20}$

In this letter, we show first that coherent surface x-ray scattering equation is mathematically equivalent to the forward scattering ptychography equation by mapping the surface height to a phase object. Then, we test the concept by simulating diffraction patterns and applying ptychographic phase retrieval algorithms to retrieve features of surface steps. We further present a proof-of-concept experiment on a Pt (111) surface where high contrast features were obtained in the phase of the reconstructed image. Although intended applications of our approach are for interfaces buried in a liquid or solid, or under extreme environments, here, we employed room-temperature atmospheric inert gas environments for the proof-of-concept experiment. Finally, we discuss further improvements to be considered in future experiments.

Let us consider a crystal surface defined by a height function, $h\left(x_{s}, y_{s}\right)$, where $s$ denotes the sample coordinate system. If a uniform illumination is assumed, the expression for the surface height function is already well known from the equations by Sinha et al..$^{21}$ and by Vartanyants et al. ${ }^{10}$ When we consider realistic non-uniform illuminations, we must include explicitly the complex-valued probe function, $p\left(x_{s}, y_{s}\right)$. Then, the diffraction amplitude for the geometry defined in Figure 1(a) 


$$
A\left(x_{c}, y_{c}\right) \propto\left|\frac{1}{q_{z s}} \iint_{s} \mathrm{~d} x_{s} \mathrm{~d} y_{s}\left\{p\left(x_{s}, y_{s}\right) e^{-i q_{z s} h\left(x_{s}, y_{s}\right)}\right\} e^{-i\left(q_{x s} x_{s}+q_{y s} y_{s}\right)}\right|
$$

and the CCD pixel position, $\left(x_{c}, y_{c}\right)$, can be expressed by $\boldsymbol{q}_{s}$ using the geometric relation, as shown in Figure 1(b), $\left(q_{x s}, q_{y s}, \delta q_{z s}\right)=\frac{4 \pi \Delta}{\lambda L}\left(x_{c}, y_{c} \sin \theta, y_{c} \cos \theta\right)$, where $\Delta$ is the pixel size and $L$ is the sample-CCD distance with $\theta$ being the incident angle of x-rays. The center of the CCD, $\boldsymbol{x}_{\mathrm{c}}=(0,0)$, is placed at $\boldsymbol{q}=\left(0,0, q_{\mathrm{zs}}\right)$ for the specular reflection condition. Note here that Eq. (1) shows that the CCD image depends only on $\left(q_{\mathrm{xs}}, q_{\mathrm{ys}}\right)$, therefore, $\left(x_{\mathrm{c}}, y_{\mathrm{c}}\right)$. From examination of Eq. (1), the diffraction from the height variation on a crystal surface in reflection mode is identical to the diffraction from a two-dimensional pure phase object in transmission geometry, except for the $\sin \theta$ factor. This similarity enables us to adopt ptychographic algorithms developed for the transmission geometry to the reflection geometry.

We have checked the validity of Eq. (1) in the ptychographic reconstruction algorithm using simulated diffraction patterns from an artificial two-dimensional (2D) surface with monoatomic height variations. We calculated diffraction patterns in the far field centered around the specular beam, based on the path length differences from the source to a detector placed perpendicular to the reflected beam at an angle close to an anti-Bragg condition. Ptychographic reconstructions of the diffraction patterns were done using a difference map algorithm with simultaneous probe retrieval. $^{22,23}$ In this zero-error simulation, the ptychographic reconstruction converges quickly and is very reproducible in reconstructing the probe and object, proving that Eq. (1) and the retrieval algorithm are valid at least for sufficiently strong intensity. ${ }^{24}$

For surface diffraction experiments, platinum, face centered cubic with lattice constant $\mathrm{a}=0.392 \mathrm{~nm}$, was chosen because of its clean surface structure and strong scattering. Atomic force microscopy was used to confirm single atomic steps after surface annealing and before x-ray experiments. Coherent $\mathrm{x}$-ray scattering experiments were performed at beam line 34-ID-C of the Advanced Photon Source (APS) at Argonne National Laboratory. $9 \mathrm{keV}$ x-rays from an undulator source were slit down to $30(\mathrm{~h}) \times 50(\mathrm{v}) \mu \mathrm{m}^{2}$ at
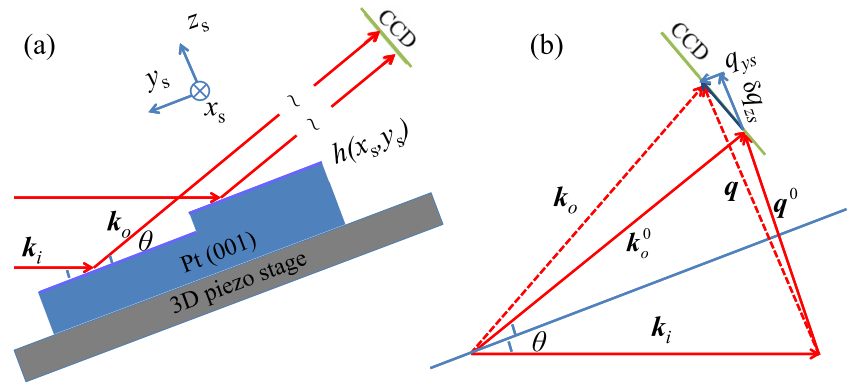

FIG. 1. Real-space (a) and reciprocal-space (b) scattering geometries are shown. A surface, described by $h\left(x_{\mathrm{s}}, y_{\mathrm{s}}\right)$, with an atomic step is schematically shown in (a). The index "s" in $\left(x_{\mathrm{s}}, y_{\mathrm{s}}, z_{\mathrm{s}}\right)$ and $\left(q_{x \mathrm{~s}}, q_{y \mathrm{~s}}, q_{z \mathrm{~s}}\right)$ indicates surface coordinate system. (b) Each CCD pixel represents a $\boldsymbol{k}_{\mathbf{o}}$, thus a scattering wave vector $\boldsymbol{q}=\boldsymbol{k}_{\mathbf{o}}-\boldsymbol{k}_{\mathbf{i}}=\boldsymbol{q}^{0}+\delta \boldsymbol{q}_{\mathrm{zs}}+\boldsymbol{q}_{\mathrm{ys} .} \boldsymbol{k}_{o}^{0}$ points to the CCD center for the specular reflection of $\boldsymbol{q}^{0}$. approximately $30 \mathrm{~m}$ distance from the source and focused with Kirkpatrick-Baez (KB) mirrors to a beam size of about $1 \mu \mathrm{m}$. A confocal laser-scanning microscope (LEXT, OLS3100) was aligned with the center of the goniometer to provide in situ guide in terms of direction of step edges during $\mathrm{x}$-ray experiments. The platinum single crystal was mounted on a three-dimensional (3D) translational piezo stage with nanometer position accuracy and scanned for the ptychographic data. The experimental diffraction patterns were collected with an Andor iKon-M SY CCD with $1024 \times 102413 \times 13 \mu \mathrm{m}$ pixels. Coherent diffraction patterns from a test object were collected at the beginning of the experiment in transmission geometry and used to reconstruct the probe using ptychography to have a good initial guess for the subsequent surface ptychography experiment. ${ }^{24}$

The platinum crystal surface was set at an angle $\theta=15.3^{\circ}$ for $\mathrm{Pt}\left(\begin{array}{lll}0 & 0 & 1.5\end{array}\right)$, and the $\mathrm{CCD}$ was placed at $543 \mathrm{~mm}$ downstream from the sample at an angle of $2 \theta$. This $\boldsymbol{q}$ was chosen instead of ( $\left.\begin{array}{lll}0 & 0 & 1\end{array}\right)$, where the intensity was too low to obtain statistically significant signal-to-noise ratios. In Figure 2(a), we show diffraction patterns measured at the Pt $(0,0,1.5)$ from different areas on the Pt surface. The diffraction patterns change markedly from one area to another. The patterns are generally elongated in a direction indicating the average step orientation within the illuminated area. We chose an area with a sufficiently interesting without much change in the elongation direction. The several diffraction patterns from overlapping probe positions are shown in Figure 2(b). The slant direction of the diffraction patterns is

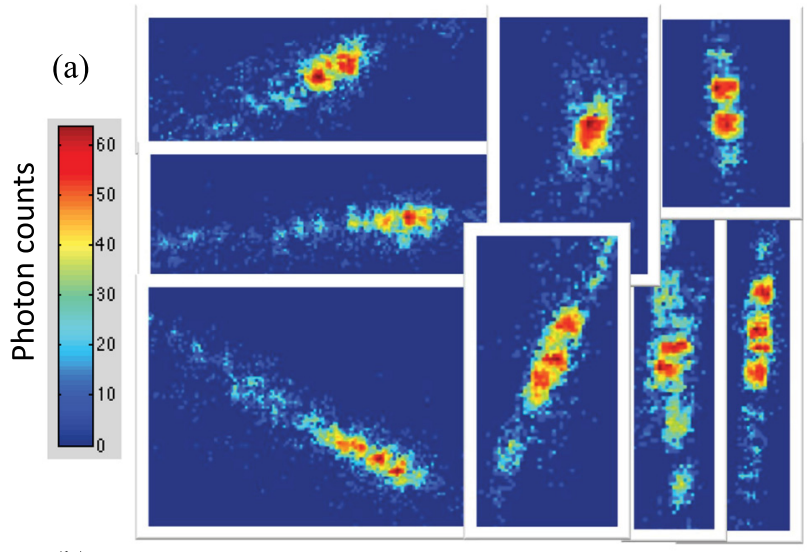

(b)

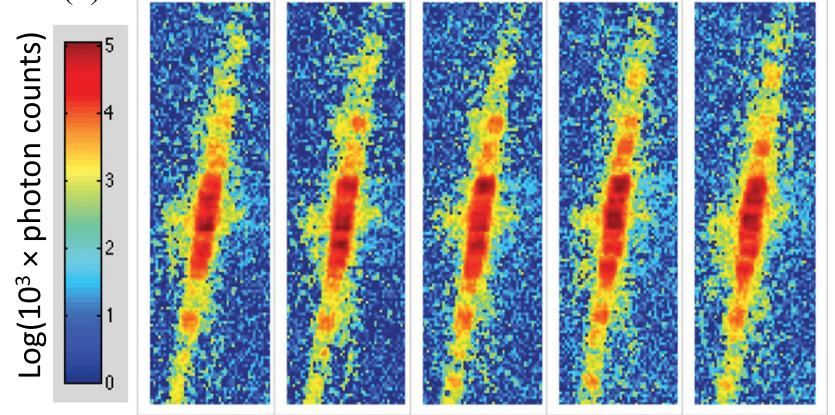

FIG. 2. (a) Diffraction patterns are shown from several randomly picked areas over $\sim 100 \mu \mathrm{m}$ surface area. The various elongated directions indicate the step directions. The color scale bar indicates the photon counts per min. (b) A set of five contiguous diffraction patterns from the area used for image reconstruction. The scale bar in this case indicates the logarithm of CCD readouts (1000 per photon) per min. 
approximately maintained within the data set, suggesting that the step directions are not changing much over the scanned area of a few microns. However, the detailed speckle patterns are different (shown in a log scale) indicating the step distribution changes.

For ptychographic experiments, coherent diffraction patterns were collected without changing the diffractometer angles at 83 scan positions for $100 \mathrm{~s}$ each on a grid of concentric circles with $0.3 \mu \mathrm{m}$ radial and $0.5 \mu \mathrm{m}$ angular steps on the $x_{l}-y_{l}$ plane, which covers an ellipsoidal area of $3 \mu \mathrm{m} \times 11.4 \mu \mathrm{m}$ on the sample surface. An area of $248 \times 248$ pixels on the detector was used around the center of the diffraction patterns.

The phase reconstruction is shown in Figure 3(a), showing features of high phase contrast. There are several indications that the reconstruction of the Pt surface did not work as


FIG. 3. Ptychographic reconstruction of Pt (111) surface. (a) Reconstructed object phase (axes not to scale). (b) Reconstructed object amplitude. (c) Reconstructed probe amplitude. (d) Reconstructed complex-valued probe with hue indicating phase and saturation indicating amplitude. (e) Probe propagation in vacuum with respect to sample position at $z_{l}=0$. well as the test specimen in transmission geometry. ${ }^{24}$ First of all, we would expect a few or no structure in the amplitude. However, we observe a remarkable structure in the amplitude image, as shown in Figure 3(b) correlated to the structure revealed in the phase image (a). Second, the reconstructed image has some vortices, which are singularities with zero amplitude and phase changing from $-\pi$ to $\pi$ around this point. Phase vortices can be physically meaningful for dislocation singularities. ${ }^{25}$ However, vortices are also sometimes observed as artifacts in ptychographic reconstructions for objects with many waves in the phase, as may be the case in atomic step reconstructions. ${ }^{19}$ This is supported by the fact that vortices appear at different positions on the image for different iterations number in the difference map reconstruction. Therefore, we attribute the vortices in our reconstructions to an incomplete convergence of the algorithm. Finally, the reconstructed probe shown in Figures 3(c) and $3(\mathrm{~d})$ is not identical to the initial probe and exhibits some of the specimen structures, which is an indication that the algorithm did not succeed to separate completely the object from the probe, possibly due to change in the probe occurring over the long measurement time.

We nevertheless retrieve a clear structure in the object which is reproducible, as tested by examining the result after different iterations of the difference map algorithm (see the supplementary material ${ }^{24}$ ). Moreover, the probe also converges to a focused beam with fringes, as shown in Figures 3(c)-3(e), as expected from the initial input probe. All this makes us confident that some main features on the reconstructed object, such as the sharp phase steps indicated in Figure 4, are real features arising from the surface topography of the sample. In Figure 4(b), we show the phase image of the rectangular region marked in Figure 3(a) to a same scale for $x_{s}$ and $y_{s}$. The pixel size of the reconstructed image

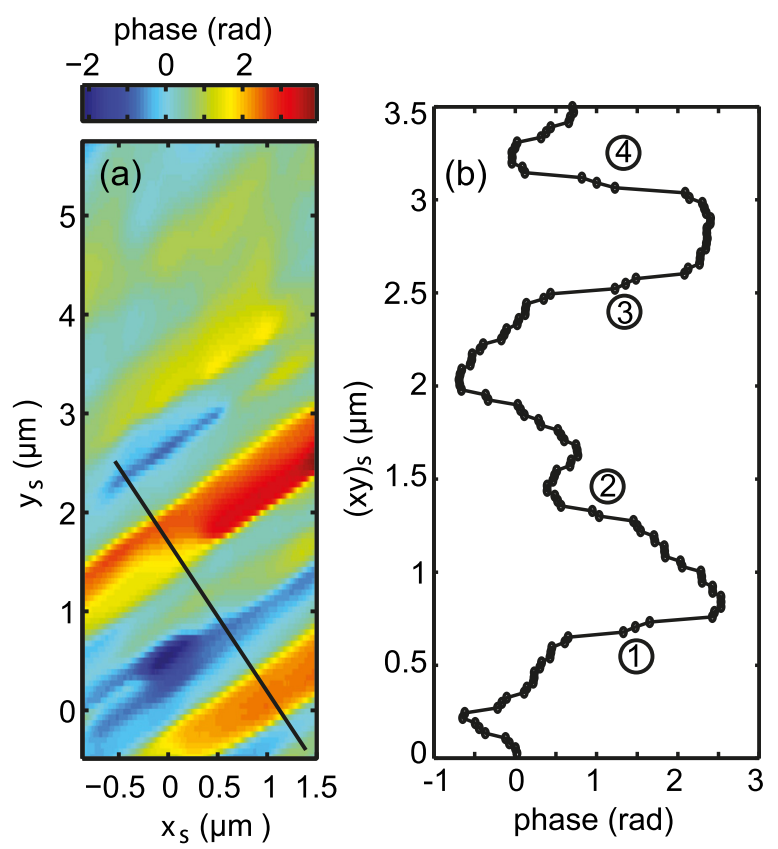

FIG. 4. (a) Region of interest indicated in Figure 3(a) with dashed lines is redrawn to a same scale for $x_{\mathrm{s}}$ and $y_{\mathrm{s}}$ after the phase has been unwrapped in order to visualize the structure better. (b) Phase profile indicated by a line in (a) reveals sharp steps indicated by numbers $1-4$. Note that the steps are in a few angstroms, while the lateral lengths are in microns. 
is $15 \mathrm{~nm}$ along $x_{s}$ but, due to the incidence angle of the beam on the sample surface, the pixel size on the $y_{s}$ direction is $57 \mathrm{~nm}$. In this image, the phase has been unwrapped for better visualization of the phase structure. ${ }^{26}$ Note that some of the phase jumps observed in (a) are due to phase wrapping within the range between $-\pi$ and $\pi$. In Figure 4(b), we show the phase profile indicated by the black continuous line in Figure 4(a), where we clearly observe structures with sharp phase steps of about $2 \mathrm{rad}$, as seen in the features on Figure 4(c) labeled as 1, 3, and 4, and of about $1 \mathrm{rad}$, labeled as 2 . This structure could arise from atomic steps on the sample surface, although the observed step sizes differ from the expected phase steps of $3 \pi / 2=4.7 \mathrm{rad}$. In this diagonal cut through the image, the pixel size is about $27 \mathrm{~nm}$, and the apparent resolution is estimated to be about $100 \mathrm{~nm}$ from the width of the sharp steps.

The phasing algorithms of CDI often fail in the cases of nanocrystals with large phase jumps ${ }^{27}$ or nanowires with stacking faults. ${ }^{28}$ Spatial resolution in ptychography is limited by the extension of the diffraction patterns measureable with sufficient signal-to-noise ratio and by position accuracy of the specimen with respect to the probe. We find that the coherent surface $\mathrm{x}$-ray scattering signal near anti-Bragg is typically limited to $\sim 100$ counts at the pixel of the maximum intensity by counting for a minute. The measurements longer than a minute per position did not improve the reconstructed images as the data are prone to source and probe instabilities, thermal drifts, and mechanical instabilities of sample stages. However, ptychography is a robust technique and expect to produce reconstructed images representing the real images even though resolutions can be compromised because of the low photon counts. We show that the main feature of the star pattern is indeed reproduced even when limited photon counts ( $\sim 100$ counts maximum), comparable to the experimental photon counts, are used in the simulation. ${ }^{24} \mathrm{We}$ expect the photon counts will significantly increase in future $x$-ray sources. Meanwhile, incorporation of various corrections such as probe position refinement ${ }^{29}$ or taking partial coherence of the illumination into account ${ }^{30,31}$ could still be attempted to try to improve the reconstruction of surface step structure. Improvements in mechanical stabilities of sample translation stage and diffractometer will also help to further enhance the reconstructed images.

In conclusion, we have proposed to combine $\mathrm{x}$-ray ptychography and crystal truncation rod for surface imaging. Near Anti-Bragg condition where the coherent diffraction intensity is sensitive to surface heights, we show that the surface height function can be reformulated as an object with large phase variations in a transmission ptychography case, thereby elucidating that the surface height profile is obtainable from the ptychographic reconstruction of the phase object. It is shown in simulations ${ }^{24}$ that atomic step heights can be quantitatively imaged with atomic-level sensitivity. We also show the feasibility of the ptychographic reconstruction from experimental diffraction data collected from $\mathrm{Pt}$ (001) surfaces. We reconstructed images with large phase jumps, which we tentatively attribute to images of monoatomic surface steps. With improved data acquisition strategies, phase retrieval algorithms and further developed instrumentation for high-accuracy positioning on the nanoscale, ${ }^{32}$ we expect this approach to have a potential for high-resolution in situ imaging of surface structures in reactive and extreme environments and buried interfaces.

We thank Jon Hiller for assistance in Electron Microscopy Center and Joan Vila-Comamala for providing the test pattern. The work at Materials Science Division (C.Z., A.B., Y.L., and H.Y.) was supported by the Materials Sciences and Engineering Division and the work at the Advanced Photon Source (R.H., R.X., and X.H.) and the Electron Microscopy Center by the Scientific User Facilities Division, of the U.S. Department of Energy, Office of Basic Energy Sciences, under Contract No. DE-AC0206CH11357. The work was also in part performed at Swiss Light Source (A.D. and A.M.) and Rochester Institute of Technology (M.S.P.), and Safarik University (V.K.). The work at Safarik University was supported by Slovak Grant VEGA 1/0782/12 and ERDF EU Grant under Contract No. ITMS 26220120005.

${ }^{1}$ G. Ertl, Science 254, 1750 (1991).

${ }^{2}$ A. Wieckowski and M. Neurock, Adv. Phys. Chem. 2011, 1.

${ }^{3}$ G. Binning, H. Rohrer, C. Gerber, and E. Weibel, Phys. Rev. Lett. 49, 57 (1982).

${ }^{4}$ F. Giessibl, Rev. Mod. Phys. 75, 949 (2003).

${ }^{5}$ C. Leslie, E. Landree, C. Collazo-Davila, E. Benque, and L. D. Marks, Microsc. Res. Tech. 46, 160 (1999).

${ }^{6}$ I. K. Robinson, Phys. Rev. B 33, 3830 (1986).

${ }^{7}$ J. Wang, A. J. Davenport, H. S. Isaacs, and B. M. Ocko, Science $\mathbf{2 5 5}$, 1416 (1992).

${ }^{8}$ V. Komanicky, H. Iddir, K.-C. Chang, A. Menzel, G. Karapetrov, D. Hennessy, P. Zapol, and H. You, J. Am. Chem. Soc. 131, 5732 (2009).

${ }^{9}$ A. Menzel, K.-C. Chang, V. Komanicky, Y. V. Tolmachev, A. V. Tkachuk, Y. S. Chu, and H. You, Phys. Rev. B 75, 035426 (2007).

${ }^{10}$ I. A. Vartanyants, J. A. Pietney, J. L. Libbert, and I. K. Robinson, Phys. Rev. B 55, 13193 (1997).

${ }^{11}$ H. You, M. Pierce, V. Komanicky, A. Barbour, and C. Zhu, Electrochim. Acta 82, 570 (2012).

${ }^{12}$ I. K. Robinson, J. L. Libbert, I. A. Vartanyants, J. A. Pitney, D. M. Smilgies, D. L. Abernathy, and G. Grubel, Phys. Rev. B 60, 9965 (1999).

${ }^{13}$ T. Sun, Z. Jiang, J. Strzalka, L. Ocola, and J. Wang, Nat. Photonics 6, 586 (2012).

${ }^{14}$ P. Fenter, C. Park, Z. Zhang, and S. Wang, Nat. Phys. 2, 700 (2006).

${ }^{15}$ J. M. Rodenburg and H. M. L. Faulkner, Appl. Phys. Lett. 85, 4795 (2004).

${ }^{16}$ M. Dierolf, A. Menzel, P. Thibault, P. Schneider, C. M. Kewish, R. Wepf, O. Bunk, and F. Pfeiffer, Nature 467, 436-439 (2010).

${ }^{17}$ P. Godard, G. Carbone, M. Allain, F. Mastropietro, G. Chen, L. Capello, A. Diaz, T. H. Metzger, J. Stangl, and V. Chamard, Nat. Commun. 2, 568 (2011).

${ }^{18}$ S. O. Hruszkewycz, M. V. Holt, C. E. Murray, J. Bruley, J. Holt, A. Tripathi, O. G. Shpyrko, I. McNulty, M. J. Highland, and P. H. Fuoss, Nano Lett. 12, 5148 (2012).

${ }^{19}$ M. S. Pierce, D. C. Hennessy, K. C. Chang, V. Komanicky, J. Strzalka, A. Sandy, A. Barbour, and H. You, Appl. Phys. Lett. 99, 121910 (2011).

${ }^{20}$ G. Prevot and B. Croset, Phys. Rev. B 74, 235410 (2006).

${ }^{21}$ S. K. Sinha, E. B. Sirota, S. Garoff, and H. B. Stanley, Phys. Rev. B 38, 2297 (1988)

${ }^{22}$ P. Thibault, M. Dierolf, A. Menzel, O. Bunk, C. David, and F. Pfeiffer, Science 321, 379 (2008).

${ }^{23}$ P. Thibault, M. Dierolf, O. Bunk, A. Menzel, and F. Pfeiffer, Ultramicroscopy 109, 338 (2009).

${ }^{24}$ See supplementary material at http://dx.doi.org/10.1063/1.4914927 for simulations, preliminary measurements, and reconstruction details.

${ }^{25}$ Y. Takahashi, A. Suzuki, S. Furutaku, K. Yamauchi, Y. Kohmura, and T. Ishikawa, Phys. Rev. B 87, 121201 (2013).

${ }^{26}$ R. M. Goldstein, H. A. Zebken, and C. L. Werner, Radio Sci. 23, 713-720, doi:10.1029/RS023i004p00713 (1988).

${ }^{27}$ I. K. Robinson, J. Phys. Soc. Jpn. 82, 021012 (2013). 
${ }^{28}$ V. Favre-Nicolin, F. Mastropietro, J. Eymery, D. Camacho, Y. M. Niquet, B. M. Borg, M. E. Messing, L.-E. Wernersson, R. E. Algra, E. P. A. M. Bakkers, T. H. Metzger et al., New J. Phys. 12, 035013 (2010).

${ }^{29}$ F. Zhang, I. Peterson, J. Vila-Comamala, A. Diaz, F. Berenguer, R. Bean, B. Chen, A. Menzel, I. K. Robinson, and J. M. Rodenburg, Opt. Express 21, 13592 (2013).
${ }^{30}$ L. W. Whitehead, G. J. Williams, H. M. Quiney, D. J. Vine, R. A. Dilanian, S. Flewett, and K. A. Nugent, Phys. Rev. Lett. 103, 243902 (2009).

${ }^{31} \mathrm{P}$. Thibault and A. Menzel, Nature 494, 68 (2013).

${ }^{32}$ M. Holler, A. Diaz, M. Guizar-Sicairos, P. Karvinen, E. Färm, E. Härkönen, M. Ritala, A. Menzel, J. Raabe, and O. Bunk, Sci. Rep. 4, 3857 (2014). 